\title{
Spatial Risk Premium on Weather Derivatives and Hedging Weather Exposure in Electricity
}

\author{
Wolfgang Karl Härdle* \\ Maria Osipenko*
}

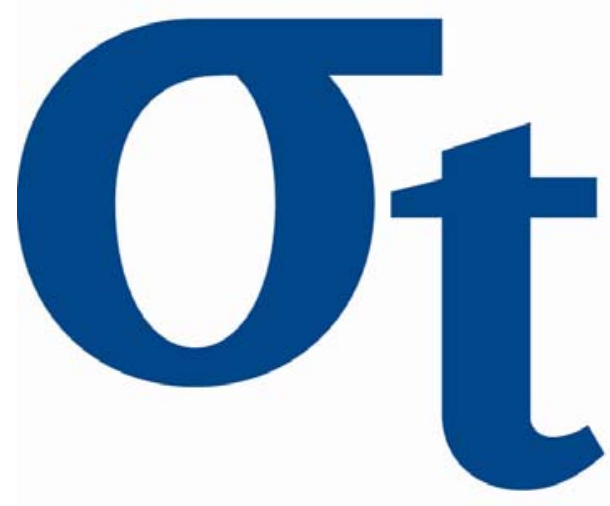

* Humboldt-Universität zu Berlin, Germany 


\title{
Spatial Risk Premium on Weather Derivatives and Hedging Weather Exposure in Electricity*
}

\author{
Wolfgang Karl Härdleł
}

\begin{abstract}
Due to dependency of energy demand on temperature, weather derivatives enable the effective hedging of temperature related fluctuations. However, temperature varies in space and time and therefore the contingent weather derivatives also vary. The spatial derivative price distribution involves a risk premium. We examine functional principal components of temperature variation for this spatial risk premium. We employ a pricing model for temperature derivatives based on dynamics modelled via a vectorial Ornstein-Uhlenbeck process with seasonal variation. We use an analytical expression for the risk premia depending on variation curves of temperature in the measurement period. The dependence is exploited by a functional principal component analysis of the curves. We compute risk premia on cumulative average temperature futures for locations traded on CME and fit to it a geographically weighted regression on functional principal component scores. It allows us to predict risk premia for nontraded locations and to adopt, on this basis, a hedging strategy, which we illustrate in the example of Leipzig.
\end{abstract}

Keywords: risk premium, weather derivatives, Ornstein-Uhlenbeck process, functional principal components, geographically weighted regression

JEL classification: C01; C31

*This research was supported by Deutsche Forschungsgemeinschaft through the SFB 649 /Economic Risk $/$.

${ }^{\dagger}$ C.A.S.E. - Center for Applied Statistics \& Economics, Humboldt-Universität zu Berlin, Germany

${ }^{\ddagger}$ Graduate Institute for Statistics, National Central University, Jhongli, Taiwan

${ }^{\S}$ Corresponding author. Email: maria.osipenko@wiwi.hu-berlin.de, Address: CASE, School of Business and Economics, Humboldt-Universiät zu Berlin, Unter den Linden 6, 10099, Germany. 


\section{Introduction}

Weather events play an important role for industries with profits depending on temperature or other weather conditions. Examples of such industries are energy, tourism and agricultural sectors. As a result a market for trading on temperature events has recently emerged. The traded financial contracts allowing for the transfer of weather risks are called weather derivatives. The value of those contracts depends on a certain weather event, which is often the average temperature.

This kind of financial instrument in OTC markets was introduced in 1997 for the first time according to Considine (2000). Exchange weather derivatives (WD) were lauched by the CME in 1999. Since then the trading volume has had an upward trend and geographic coverage of underlying weather indices has expanded as well. Currently there is a wide range of products available on the CME. Besides WDs based on temperature indices, there are contracts on hurricane, frost, snowfall and rainfall (see http://www.cmegroup.com/trading /weather/). Temperature based derivatives are the most popular and have the widest geographical range. Contracts for 24 cities in the US, six in Canada, 11 in Europe, three in Australia and three in Japan are available for trading to date.

Basic types of contracts are calender period futures and options on heating degree days (HDD), cooling degree days (CDD), cumulative average temperature (CAT) and the cumulative total of 24-hour average temperatures (C24AT). A single day calculation of the corresponding temperature indices is carried out the following way: HDD index is computed as the maximum of zero and $65^{\circ} \mathrm{F}\left(18^{\circ} \mathrm{C}\right)$ minus the average temperature of the day, CDD correspondingly as the maximum of zero and the average temperature minus $65^{\circ} \mathrm{F}\left(18^{\circ} \mathrm{C}\right)$. HDD's and CDD's are then accumulated over the corresponding contract period. CAT and C24AT cumulate daily average temperature (average of maximum and minimum temperature) and 24-hour average temperature of each day respectively. Trading months (seasons) for CDD and CAT contracts are April to October, for HDD October through to April and for C24AT all months of the year.

A CME traded future contract on such indices corresponds to a swap, such that one party gets paid if the realised index value is greater than the strike (price of the contract) and the other party benefits if the index value is below. The notional value of one contract is 20 USD (20 EUR, 20 GBP or 2500 JPY) per index point, in which the contracts are also quoted. 
The futures market for temperature indices is primarily driven by the energy sector, which recognised the hedging power of WD in reducing earnings fluctuations due to the weather dependent demand, see Perez-Gonzalez and Yun (2010). Although, the European weather market is behind the US, primarily by trading volume, it is growing and the recent hot summer activated trading with European CAT futures. The trading volume for $\mathrm{Au}-$ gust 2010 was $2.6 \mathrm{Mln}$. GBP for UK trades and 1.71 Mln. EUR for European trades (as reported by http://www.trading-risk.com/european-weathertrading-flourishes-in-the-heat).

Figure 1 illustrates the estimated dependence between German electricity demand and CAT monthly from 199601 to 201009 (data from EUROSTAT). Using a semiparametric partial linear model as in Akdeniz-Duran et al. (2011) one sees a nonlinear relationship. The described effect of temperature on demand justifies hedging potential of weather futures. Similar observations on aggregate energy intensity and temperature indices can be found in Metcalf (2008) and with respect to natural gas consumption and HDD in Asche et al. (2008).

However, geographical coverage of the European market for WD is still small. There are 11 cities on which temperature derivatives are traded. For many important locations of weather dependent industries no weather derivatives are traded. The need to calculate prices for those regions therefore arises.

Many authors investigated the problem of finding an appropriate pricing model for WD. The main stream approaches can be divided in those that derive prices purely from temperature dynamics and those that take into account utility and profit functions of weather market participants. Dornier and Querel (2000) and Alaton et al. (2002) fitted an Ornstein-Uhlenbeck process to the temperature observations and demonstrated effect of mean, variance and market price of risk on CDD and HDD option prices following the first approach. Campbell and Diebold (2005) applied an autoregressive process of a higher order to model deseasonalised temperature and observed seasonality in temperature variation. Benth et al. (2007) and Härdle and López Cabrera (2011) propose to model temperature dynamics with a deterministic seasonal component and a higher order continuous autoregressive process with seasonal variation. They also derive arbitrage free prices for future contracts on CDD and CAT indices. 


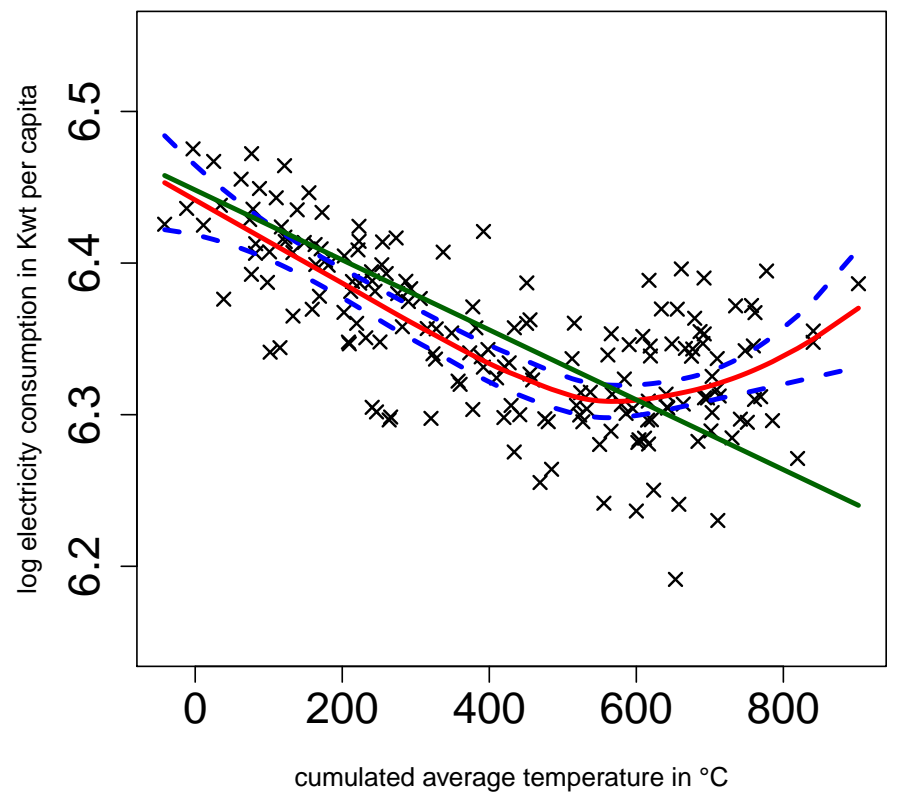

Figure 1: The impact of cumulated average temperature, estimated assuming linear and nonlinear underlying dependence from Akdeniz-Duran et al. (2011). Log electricity consumption against monthly cumulated temperature index (circles) and the estimated linear (green) and nonparametric (red) effect of temperature with $95 \%$ confidence intervals (dashed).

For the approaches which impute utility and profit functions of weather market participants, see for example Chaumont and Müller (2006), Horst and Müller (2007) or Lee and Oren (2009) who assume agents maximise their utility possibly dependent on weather and derive optimal pricing in a partial market clearing setting. Davis (2001) proposes a profit based pricing by marginal value.

In the current work we stick to the first data driven approach since we have trading WD data from the CME as well as temperature available and consider stochastic dynamics of temperature as a basis for pricing, avoiding thereby restrictive assumptions on utility and profit functions. Temperature based WD on the CAT index are considered, currently traded for Berlin, Amsterdam, Barcelona, Essen, London, Madrid, Paris, Rome and Stockholm. They are used to construct a spatial model for the involved risk premia. Risk premia may be then calculated for arbitrary locations using functional principal 
component scores of temperature variation. We also provide an example of a hedging strategy using CAT futures.

This paper is organised as follows. In the next section the theoretical pricing model for temperature based derivatives and spatial specification are considered. Section 3 continues with applying the theoretical model to real temperature data and computing risk premia and reports the estimation results. We give an example of hedging with WD for the city of Leipzig. Finally, we conclude and give an outook on future work.

\section{Pricing Model for Temperature Futures}

A reliable valuation procedure for a WD plays a key role in the effectiveness of transfering risk. Any pricing model for WD must be complicated by the fact that the underlying (weather) can not be traded and therefore WDs can not be replicated by long positions in the underlying and risk-free asset. Additionally, markets for WDs are not very liquid and thus they can't be replicated by other WDs.

We follow here the pricing methodology applied by Härdle and López Cabrera (2011) originally developed by Benth et al. (2007).

\subsection{Temperature Dynamics in Discrete Time}

For a pricing model for WDs, one has to find a good approximation for the evolution of the underlying, in this case - the temperature. From the observed data we construct a discrete time model:

- Let $T_{t}$ be the (average) temperature in day $t, t=1, \ldots, M$ :

$$
T_{t}=\Lambda_{t}+Y_{t},
$$

- $\Lambda_{t}$ is a deterministic seasonal function:

$$
\Lambda_{t}=a+b t+\sum_{i=1}^{s} c_{2 i} \cos \{2 i \pi t / 365\}+c_{2 i+1} \sin \{2 i \pi t / 365\}
$$

- $Y_{t}$ is a $p$-order autoregressive process

$$
Y_{t}=\alpha_{0}+\sum_{i=1}^{p} \alpha_{i} Y_{t-i}+\sigma_{t} \varepsilon_{t}
$$


- $\varepsilon_{t}$ is white noise and $\sigma_{t} \stackrel{\text { def }}{=} \sigma(t)$ is the smooth function of time representing variation of temperature on each day of the Julian calender.

The seasonality $\Lambda_{t}$ in (2) is assumed to have a periodical form with time trend. The parameter $a$ represents average temperature over the years and $b$ stands for the deterministic time trend. The set of parameters $c=\left\{c_{i}\right\}_{i=1}^{s}$ captures seasonal/periodical dynamics of temperature. Variation of temperature is assumed to follow a seasonal pattern as well. The number of Fourier series terms $s$ is typically region/climate specific. In a particular application $s$ can be chosen by Schwarz information criteria.

The stochastic process $Y_{t}$ in (3) is modelled by an autoregressive process. However, before one can proceed, the resulting series $\left\{Y_{t}\right\}_{t=1}^{T}$ has to be tested for stationarity. For this purpose we apply two tests: Augmented DickeyFuller (ADF) test for a unit root and KPSS test for stationarity. The order of the appropriate autoregressive process is chosen via Box-Jenkins analysis, Box and Jenkins (1970) and BIC, Hurvich and Tsai (1989).

Since there is seasonal variation in the residuals, the process in (3) is heteroscedastic and we have to account for it in the model. Therefore we group the residuals in 365 groups, so that each group represents residuals of the same day over all years. Then we compute the variation of each day and smooth it with Fourier series.

\subsection{Continuous Time Model for Temperature Dynam- ics}

In order to apply a pricing model it is convenient to switch to continuous time and transfer our obtained discrete time temperature dynamics for $T_{t}$ to a continuous time process $T(t)$. For that purpose we use the continuous time autoregressive model $(\mathrm{CAR}(p))$ with seasonal variation described by Benth et al. (2007).

A CAR $(p)$ model can be represented in terms of a state-space $p$-dimensional Ornstein - Uhlenbeck process. Then, dynamics of temperature $T(t)$ as continuous function of time can be rewritten:

$$
T(t)=\Lambda(t)+X_{1}(t)
$$

where $\Lambda(t)$ is a continuous time version of $\Lambda_{t}$ in (2), $X_{k}(t)$ is the $k$-th coordinate of the stochastic process $X(t)$, defined by the vectorial Ornstein- 
Uhlenbeck differential equation:

$$
d X(t)=A X(t) d t+\mathbf{e}_{p} \sigma(t) d B(t)
$$

with $\mathbf{e}_{k}$ denoting $k$-th unit vector in $\mathbb{R}^{p}, \sigma(t)>0$ is a real valued and integrable function and $A$ is a $(p \times p)$-matrix, defined as:

$$
A=\left(\begin{array}{ccccc}
0 & 1 & 0 & \ldots & 0 \\
0 & 0 & 1 & \ldots & 0 \\
\vdots & \vdots & \vdots & \ddots & \vdots \\
0 & 0 & 0 & \ldots & 1 \\
-\tilde{\alpha}_{p} & -\tilde{\alpha}_{p-1} & -\tilde{\alpha}_{p-2} & \ldots & -\tilde{\alpha}_{1}
\end{array}\right)
$$

The explicit solution to this stochastic differential equation is:

$$
X(s)=\exp \{A(s-t) x\}+\int_{t}^{s} \exp \{A(s-u)\} \mathbf{e}_{p} \sigma(u) d B(u) .
$$

For details see Gillespie (1996). Stationarity of such a process is ensured by the negative real parts of all eigenvalues of $A$, see below. To identify the $\operatorname{CAR}(p)$ model associated with the fitted $\operatorname{AR}(p)$ model we use Euler approximations of the $X_{t}$ dynamics with time step 1 and obtain for $X_{1, t}$ from (4),

$$
\text { for } \begin{aligned}
p=1: X_{1}(t+1)= & \left(1-\tilde{\alpha}_{1}\right) X_{1}(t), \\
p=2: X_{1}(t+2)= & \left(2-\tilde{\alpha}_{1}\right) X_{1}(t+1)+\left(\tilde{\alpha}_{1}-\tilde{\alpha}_{2}-1\right) X_{1}(t), \\
p=3: X_{1}(t+3)= & \left(3-\tilde{\alpha}_{1}\right) X_{1}(t+2)+\left(2 \tilde{\alpha}_{1}-\tilde{\alpha}_{2}-3\right) X_{1}(t+1) \\
& +\left(-\tilde{\alpha}_{1}+\tilde{\alpha}_{2}-\tilde{\alpha}_{3}+1\right) X_{1}(t), \\
p=4: X_{1}(t+4)= & \left(4-\tilde{\alpha}_{1}\right) X_{1}(t+3)+\left(3 \tilde{\alpha}_{1}-\tilde{\alpha}_{2}-6\right) X_{1}(t+2) \\
& +\left(4+2 \tilde{\alpha}_{2}-\tilde{\alpha}_{3}-3 \tilde{\alpha}_{1}\right) X_{1}(t+1) \\
& +\left(\tilde{\alpha}_{1}+\tilde{\alpha}_{3}-\tilde{\alpha}_{4}-\tilde{\alpha}_{2}-1\right) X_{1}(t) .
\end{aligned}
$$

where $\tilde{\alpha}_{1}, \tilde{\alpha}_{2}, \tilde{\alpha}_{3}, \ldots, \tilde{\alpha}_{p}$ denote the autoregressive coefficients of the $\operatorname{CAR}(p)$ model (5) and $\alpha_{1}, \alpha_{2}, \ldots \alpha_{p}$ denote the coefficients of $\operatorname{AR}(p)$. By identification of coefficients we obtain, e.g. for $\operatorname{AR}(4)$ : $\tilde{\alpha}_{1}=4-\alpha_{1}, \tilde{\alpha}_{2}=3 \tilde{\alpha}_{1}-\alpha_{2}-6$, $\tilde{\alpha}_{3}=-3 \tilde{\alpha}_{1}+2 \tilde{\alpha}_{2}-\alpha_{3}+4$ and $\tilde{\alpha}_{4}=\tilde{\alpha}_{1}-\tilde{\alpha}_{2}+\tilde{\alpha}_{3}-\alpha_{4}-1$ parameters of the $\mathrm{CAR}(4)$ model. 


\subsection{Pricing of Temperature Futures}

An important feature of the market for temperature derivatives is its incompleteness. The underlying (temperature) is not tradeable and the derivatives can not therefore be replicated. Although the markets for temperature derivatives are incomplete, their prices must be arbitrage-free, since they are tradeable assets. Therefore we assume that a pricing measure $Q=Q_{\theta(t)}$ exists and can be parametrized via Girsanov's theorem, see Øksendal (2000) by $\theta(t)$, the time varying market price of risk (MPR).

$$
F\left(t, \tau_{1}, \tau_{2}\right)=\mathrm{E}^{Q_{\theta(t)}}\left[Y_{T}\left\{T(t) \mid \mathcal{F}_{t}\right\}\right]
$$

with $0 \leq t \leq T, Y_{T}\left\{T(t) \mid \mathcal{F}_{t}\right\}$ the payoff of the temperature index at $T>t$. The temperature dynamics (5) under $Q_{\theta}$ becomes:

$$
d X(t)=\left\{A X(t)+\mathbf{e}_{p} \sigma(t) \theta(t)\right\} d t+\mathbf{e}_{p} \sigma(t) d B^{\theta}(t),
$$

where $B^{\theta}(t) \stackrel{\text { def }}{=} B(t)-\int_{0}^{t} \theta(u) d u$. Explicit solution of $(8)$ is:

$$
\begin{aligned}
X(s)=\exp \{A(s-t) x\} & +\int_{t}^{s} \exp \{A(s-u)\} \mathbf{e}_{p} \sigma(u) \theta(u) d u \\
& +\int_{t}^{s} \exp \{A(s-u)\} \mathbf{e}_{p} \sigma(u) d B^{\theta}(u) .
\end{aligned}
$$

With this result we get a feasible model for pricing temperature futures. Here the unknown parameter $\theta(t)$ is the market price of risk. We can define the CAT index as:

$$
\operatorname{CAT}\left(\tau_{1}, \tau_{2}\right)=\int_{\tau_{1}}^{\tau_{2}} T(u) d u
$$

where $T(t)$ is the average temperature of day $t$. From (7) we obtain:

$$
F_{C A T\left(t, \tau_{1}, \tau_{2}\right)}=\mathrm{E}^{Q_{\theta(t)}}\left\{\int_{\tau_{1}}^{\tau_{2}} T(s) d s \mid \mathcal{F}_{t}\right\}
$$

By inserting the temperature dynamics (4) in (10) and using (9) we get for $0 \leq t \leq \tau_{1}<\tau_{2}$, see Benth et al. (2007):

$$
\begin{aligned}
F_{C A T\left(t, \tau_{1}, \tau_{2}\right)} & =\int_{\tau_{1}}^{\tau_{2}} \Lambda(u) d u+a_{t, \tau_{1}, \tau_{2}} X_{t}+\int_{t}^{\tau_{1}} \theta_{u} \sigma(u) a_{t, \tau_{1}, \tau_{2}} \mathbf{e}_{p} d u \\
& +\int_{\tau_{1}}^{\tau_{2}} \theta(u) \sigma(u) \mathbf{e}_{1}^{\top} A^{-1}\left[\exp \left\{A\left(\tau_{2}-u\right)\right\}-I_{p}\right] \mathbf{e}_{p} d u,
\end{aligned}
$$


with $a_{t, \tau_{1}, \tau_{2}}=\mathbf{e}_{1}^{\top} A^{-1}\left[\exp \left\{A\left(\tau_{2}-t\right)\right\}-\exp \left\{A\left(\tau_{1}-t\right)\right\}\right]$

With the expressions above we have the tools for pricing temperature futures. Theoretical constructs for CAT futures depend on the unknown parameter $\theta(t)$. This parameter also specifies the magnitude of the risk premia defined below for $i$ th location and $j$ th contract.

$$
R P_{i j}\left(t, \tau_{1}, \tau_{2}\right)=F_{C A T, i j}\left(\theta, t, \tau_{1}, \tau_{2}\right)-F_{C A T, i j}\left(0, t, \tau_{1}, \tau_{2}\right) .
$$

Plugging this into (11) and setting $t=\tau_{1}$ we obtain an expression for the risk premium:

$$
\begin{aligned}
R P_{i j}\left(\tau_{1}, \tau_{2}\right) & =F_{C A T, i j}\left(\theta, t=\tau_{1}, \tau_{2}\right)-\widehat{F}_{C A T, i j}\left(0, t=\tau_{1}, \tau_{2}\right)+\varepsilon_{i j} \\
& =\int_{\tau_{1}}^{\tau_{2}} \theta(u) \sigma(u) \mathbf{e}_{1}^{\top} A^{-1}\left[\exp \left\{A\left(\tau_{2}-u\right)\right\}-I_{p}\right] \mathbf{e}_{p} d u+\varepsilon_{i j} .
\end{aligned}
$$

Here $\varepsilon_{i j}$ represents the stochastic component in risk premium. Now, setting $w(t)=\mathbf{e}_{1}^{\top} A^{-1}\left[\exp \left\{A\left(\tau_{2}-t\right)\right\}-I_{p}\right] \mathbf{e}_{p}$ and $\theta_{w}(t)=\theta(t) w(t)$ we get 13).

$$
R P_{i j}\left(\tau_{1}, \tau_{2}\right)=\int_{\tau_{1}}^{\tau_{2}} \theta_{w}^{i}(u) \sigma_{i j}(u) d u+\varepsilon_{i j}
$$

On the right hand side of $(13)$ we have the inner product of two functions of time, whereas a scalar risk premium appears on the left. A functional regression with scalar response is therefore considered.

\subsection{Functional Principal Components of $\sigma_{i j}(t)$ as Input in Geographically Weighted Regression}

We deal with functional regression by extracting functional principal components of variation first and then regressing the dependent risk premium on the resulting scores, see Ramsay and Silverman (1997) for details. We decompose the $\sigma_{i j}(t)$ variation curve into an average curve $\bar{\sigma}_{i}(t)$ for the $i$ th location and deviations from it.

$$
\sigma_{i j}(t)=\left\{\sigma_{i j}(t)-\bar{\sigma}_{i}(t)\right\}+\bar{\sigma}_{i}(t)
$$


Then (13) becomes

$$
R P_{i j}=\int_{\tau_{1}}^{\tau_{2}} \theta_{w}^{i}(u) \bar{\sigma}_{i}(u) d u+\int_{\tau_{1}}^{\tau_{2}} \theta_{w}^{i}(u) \underbrace{\left\{\sigma_{i j}(u)-\bar{\sigma}_{i}(u)\right\}}_{\text {FPCA for } \sigma_{i j}} d u .
$$

The principal component scores $c_{i j k}$ for $\sigma_{i j}(t)-\bar{\sigma}_{i}(t)$ are given as:

$$
c_{i j k}=\int \xi_{i k}(t)\left\{\sigma_{i j}(t)-\bar{\sigma}_{i}(t)\right\} d t
$$

$\xi_{i k}(t)$ orthonormal eigenfunctions of the covariance operator. Having obtained the scores we regress the risk premium $R P_{i j}$ at $t=\tau_{1}$ on the first $k=1,2, \ldots, K$ scores.

$$
R P_{i j}=\beta_{0}^{i}+\int \sum_{k=1}^{K} \beta_{k}^{i} \xi_{i k}(t)\left\{\sigma_{i j}(t)-\bar{\sigma}_{i}(t)\right\} d t+\varepsilon_{i j} .
$$

This expression provides a regression setting for the sample of risk premium, with spatially varying coefficients.

With (14) one has risk premia for geographically separated locations, for which temperature derivatives are traded. We apply geographically weighted regression to model risk premia in this spatial heterogeneity setting, see Fotheringham et al. (2002). This specification introduces distance based weights, accounts for nonconstant variance over space and local heterogeneity of the spatial process. The model is specified by:

$$
W_{i}^{\frac{1}{2}} R P=W_{i}^{\frac{1}{2}} C \beta_{i}+e_{i}, \quad e_{i} i i d .
$$

with $R P=\left(R P_{1,1}, R P_{2,1}, \ldots, R P_{n, 1}, R P_{1,2}, \ldots \ldots, R P_{n, 7}\right)^{\top}$.

Here, $C=\left(\begin{array}{cccc}c_{1,1,1} & \cdots & c_{1,1, K} \\ c_{2,1,1} & \cdots & c_{2,1, K} \\ c_{n, 7,1} & \cdots & c_{n, 7, K}\end{array}\right)$ is the matrix of FPCA scores and possible dummy variables, $W_{i}, i=1, \ldots, n$ is a block diagonal weighting matrix with $w_{i}=$ $\operatorname{diag}\left[\exp \left\{-\frac{1}{2}\left(\frac{d_{i 1}}{h}\right)^{2}\right\}, \ldots, \exp \left\{-\frac{1}{2}\left(\frac{d_{i n}}{h}\right)^{2}\right\}\right]$ on the diagonal, $d_{i l}, l=1, \ldots, n$ - distances to $i$ th city, $n$-total number of locations and $K$-number of PC scores. $h$ is the decay bandwidth. The optimal bandwidth $h^{*}$ can be found by cross validation:

$$
h^{*}=\underset{h \in H}{\arg \min } \sum_{m=1}^{7 n}\left\{R P_{m}-\widehat{R P}_{\neq m}(h)\right\}^{2}
$$


In $\sqrt{16} \widehat{R P}_{\neq m}(h)$ denotes the estimated risk premium without its $m$ th value and using bandwidth $h$, see Fotheringham et al. (2002) for the deriviations. One can also consider a contract dependent bandwidth $h^{*}(j)$, however in the present application it did not differ much over $j$, so to continue we use (16) to compute $h^{*}$. In this model $\beta_{i}$ is a distance weighted sum of the other spatial coefficients, $\omega_{i}$ are the corresponding weights. The estimates are produced using:

$$
\widehat{\beta}_{i}=\left(C^{\top} W_{i} C\right)^{-1} C^{\top} W_{i} R P .
$$

We should mention that the geographically weighted regression presented above is a local linear technique to quantify spatial relationships, its results hold only locally. Therefore an out-of-sample forecast can be obtained only locally as well.

\section{Empirical Risk Premia and Hedging Weather Exposure in Electricity}

Below we present the results of the empirical analysis for European CAT futures and develop a hedging strategy for energy companies.

\subsection{Data}

For the empirical analysis we took nine European cities, for which CAT futures are traded on the CME. Temperature data were downloaded from the Deutscher Wetterdienst (Berlin and Leipzig) and Bloomberg database (Amsterdam, Barcelona, Essen, London, Madrid, Paris, Rome, Stokholm). Prices of CAT futures quoted in Bloomberg were taken to compute risk premia on traded locations. Table 1 summarises the time length and number of observations of the available datasets. The 29th February was removed from all the data.

The observations are daily average temperatures $T_{t}$ which are computed as $T_{t}=\frac{T_{t}^{\text {max }}+T_{t}^{\text {min }}}{2}$. Missing values in the data from Bloomberg were substituted by the mean of the time neighbouring observations. Relevant risk premia were computed taking prices at the first day of the measurement period or the next trading day if this day was a holiday and were averaged over the available years. 


\begin{tabular}{cccc}
\hline \hline City & First Date & Last Date & First $F_{C A T}$ Trade \\
\hline Amsterdam & 19730101 & 20101231 & 20030401 \\
Berlin & 19480101 & 20101231 & 20030401 \\
Barcelona & 19730101 & 20101231 & 20050401 \\
Essen & 19700101 & 20101231 & 20050401 \\
London & 19730101 & 20101231 & 20030401 \\
Madrid & 19730101 & 20101231 & 20050401 \\
Paris & 19730101 & 20101231 & 20030401 \\
Rome & 19730101 & 20101231 & 20050401 \\
Stockholm & 19730101 & 20101231 & 20030401 \\
Leipzig & 19730101 & 20101231 & - \\
\hline \hline
\end{tabular}

Table 1: Information about Weather Data. Source: Deutscher Wetterdienst, Bloomberg.

\subsection{Temperature Dynamics and PCA of temperature variation}

First we fit a seasonal function $\Lambda_{t}$ of the form (2) to $T_{t}$. Orders $s_{1}, s_{2}$ for the seasonality were chosen by BIC. The estimated parameters are presented in tables 2-4.

We note, that the linear time trend in $\Lambda_{t}$ is significant for all the cities on $5 \%$ level, it represents the scope of global warming effects in individual locations and have been identified to have a significant impact on energy use, see Rosenthal et al. (1995). 


\begin{tabular}{rrrrrrr}
\hline \hline & \multicolumn{2}{c}{ Amsterdam } & \multicolumn{2}{c}{ Barcelona } & \multicolumn{2}{c}{ Berlin } \\
& estimate & t.stat & estimate & t.stat & estimate & t.stat \\
\hline$a$ & 9.16 & 161.55 & 14.87 & 387.81 & 9.24 & 183.72 \\
$b$ & $1.3 \cdot 10^{-4}$ & 18.67 & $1.6 \cdot 10^{-4}$ & 32.77 & $0.4 \cdot 10^{-4}$ & 10.12 \\
$c_{1}$ & -2.60 & -64.88 & -3.38 & -124.59 & -2.86 & -80.52 \\
$c_{2}$ & -7.04 & -175.68 & -6.58 & -242.68 & -9.35 & -263.04 \\
$c_{3}$ & 0.45 & 11.25 & 1.10 & 40.43 & 0.15 & 4.10 \\
$c_{4}$ & - & - & -0.06 & -2.37 & -0.00 & -0.11 \\
$c_{5}$ & - & - & 0.03 & 1.23 & -0.05 & -1.43 \\
$c_{6}$ & - & - & -0.15 & -5.41 & 0.25 & 6.95 \\
$c_{7}$ & - & - & 0.09 & 3.32 & 0.14 & 3.88 \\
\hline \hline
\end{tabular}

Table 2: Estimated parameters of seasonality (2) for Amsterdam, Barcelona, Berlin

After removing seasonality $\Lambda_{t}$ we apply ADF and KPSS tests to check for the stationarity of $Y_{t}$. The nonstationarity of residuals could be rejected for all locations according to ADF test statistics. The KPSS test does not reject the hypothesis of stationarity in residuals as well. Since there are significant autocorrelations and partial autocorrelations in residuals we fit an $\operatorname{AR}(p)$ with order $p$ determined by BIC. Tables 5 -7 report the estimated coefficients of $\operatorname{AR}(p)$.

\begin{tabular}{rrrrrrr}
\hline \hline & \multicolumn{2}{c}{ Essen } & \multicolumn{2}{c}{ London } & \multicolumn{2}{c}{ Madrid } \\
& estimate & t.stat & estimate & t.stat & estimate & t.stat \\
\hline$a$ & 10.66 & 171.30 & 10.81 & 217.33 & 13.97 & 286.51 \\
$b$ & $0.1 \cdot 10^{-4}$ & 0.66 & $0.7 \cdot 10^{-4}$ & 11.10 & $0.9 \cdot 10^{-4}$ & 14.64 \\
$c_{1}$ & -2.32 & -52.79 & -2.48 & -70.43 & -3.30 & -95.68 \\
$c_{2}$ & -7.82 & -177.64 & -6.42 & -182.57 & -8.93 & -259.17 \\
$c_{3}$ & 0.49 & 11.21 & 0.77 & 21.85 & 1.67 & 48.46 \\
$c_{4}$ & - & - & 0.23 & 6.66 & 0.25 & 7.21 \\
$c_{5}$ & - & - & - & - & -0.19 & -5.39 \\
$c_{6}$ & - & - & - & - & -0.34 & -9.87 \\
\hline \hline
\end{tabular}

Table 3: Estimated Parameters of seasonality (2) for Essen, London, Paris 


\begin{tabular}{rrrrrrr}
\hline \hline & \multicolumn{2}{c}{ Paris } & \multicolumn{2}{c}{ Rome } & \multicolumn{2}{c}{ Stockholm } \\
& estimate & t.stat & estimate & t.stat & estimate & t.stat \\
\hline$a$ & 11.90 & 208.25 & 14.79 & 340.24 & 6.92 & 113.94 \\
$b$ & $0.3 \cdot 10^{-4}$ & 3.99 & $1.1 \cdot 10^{-4}$ & 20.98 & $0.6 \cdot 10^{-4}$ & 7.32 \\
$c_{1}$ & -2.46 & -60.90 & -3.50 & -113.90 & -3.25 & -75.72 \\
$c_{2}$ & -7.50 & -185.70 & -8.02 & -260.75 & -9.75 & -227.00 \\
$c_{3}$ & 0.71 & 17.54 & 0.93 & 30.18 & 0.26 & 5.98 \\
$c_{4}$ & - & - & 0.16 & 5.33 & 0.83 & 19.27 \\
$c_{5}$ & - & - & -0.01 & -0.29 & -0.21 & -4.94 \\
$c_{6}$ & - & - & -0.21 & -6.77 & 0.11 & 2.46 \\
$c_{7}$ & - & - & - & - & 0.26 & 6.06 \\
\hline \hline
\end{tabular}

Table 4: Estimated Parameters of seasonality (2) for Paris, Rome, Stockholm

As pointed out in the previous section, we find principal component scores by weighting $\sigma_{i j}(t)$ with the eigenfunctions of its covariance operator. The eigenfunctions for $\sigma_{i j}(t)$ are shown in Figure 4. They are to be interpreted as different positive and negative weights given deviations of temperature volatility from the mean curve $\bar{\sigma}$.

Since first three PCs already explain more than $95 \%$ of variance in the data, we discard further PCs in our analysis. The resulting three PCs are combined in a matrix of explanatory variables in spatial regression.

\begin{tabular}{lrrrrrr}
\hline \hline & \multicolumn{2}{c}{ Amsterdam } & \multicolumn{2}{c}{ Barcelona } & \multicolumn{2}{c}{ Berlin } \\
& estimate & t.stat & estimate & t.stat & estimate & t.stat \\
\hline$\alpha_{1}$ & 0.89 & 105.05 & 0.70 & 83.14 & 0.92 & 139.69 \\
$\alpha_{2}$ & -0.19 & -16.76 & 0.03 & 3.17 & -0.20 & -23.14 \\
$\alpha_{3}$ & 0.09 & 10.46 & 0.01 & 1.29 & 0.08 & 11.99 \\
$\alpha_{4}$ & - & - & 0.03 & 3.64 & - & - \\
\hline \hline
\end{tabular}

Table 5: Estimated Parameters of $\mathrm{AR}(p)$ for Amsterdam, Barcelona, Berlin

Figure 3 displays the $\hat{\sigma}(t)$ of (3) of the nine cities and their Fourier smoothed version, which will be analysed with means of principal component analysis for functional data. We now proceed with calculating $F_{C A T}$ prices based on the formula (11). Table 8 reports the observed market prices and the model prices, calculated for August 2010. To complete our spatial regression setting we estimate risk premia according to (13) and average it over the years. 

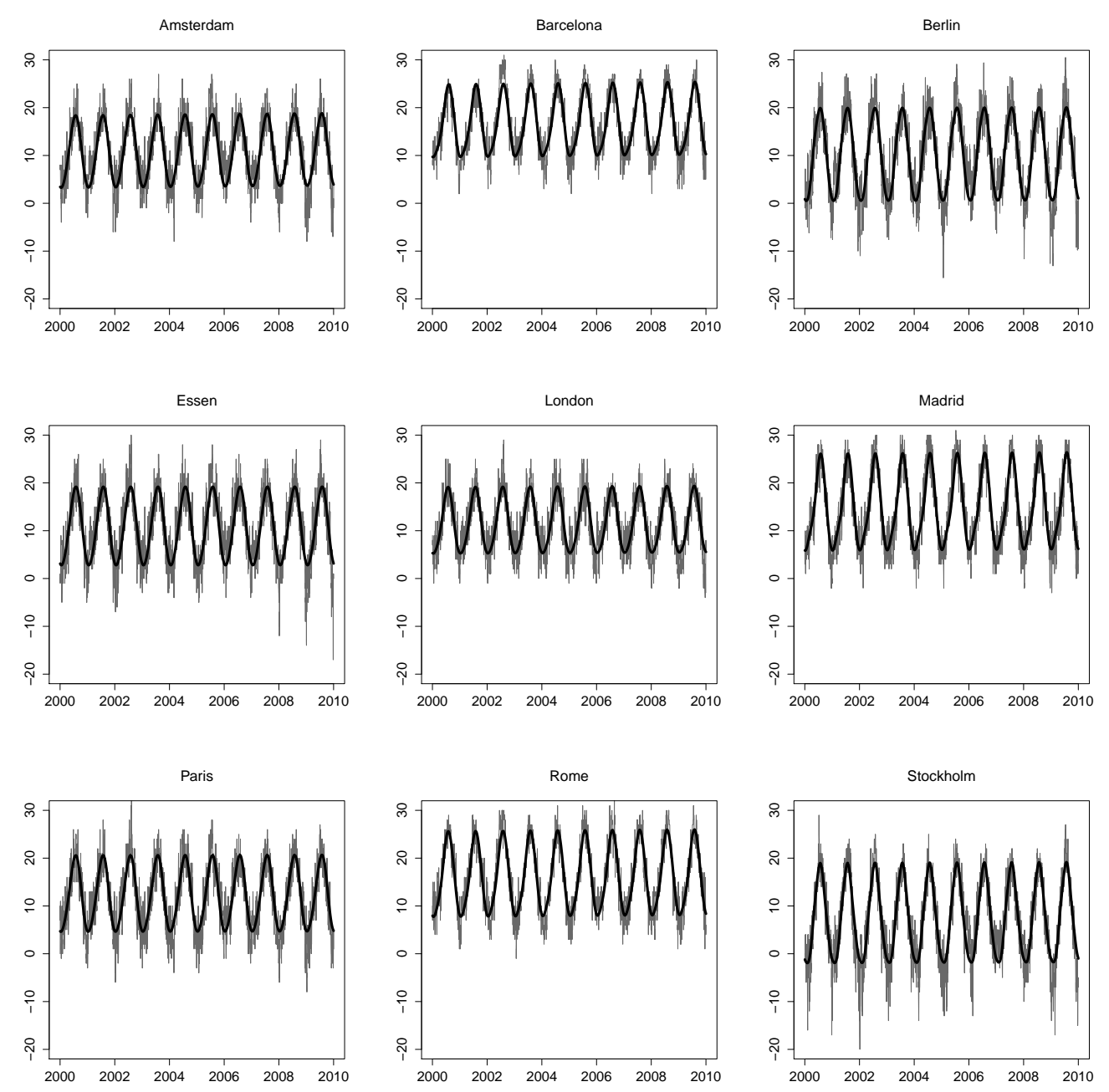

Figure 2: Daily temperature observations of the last 10 years and fitted seasonality.

\begin{tabular}{lrrrrrr}
\hline \hline & \multicolumn{2}{c}{ Essen } & \multicolumn{2}{c}{ London } & \multicolumn{2}{c}{ Madrid } \\
& estimate & t.stat & estimate & t.stat & estimate & t.stat \\
\hline$\alpha_{1}$ & 0.88 & 104.21 & 0.79 & 92.62 & 0.78 & 92.22 \\
$\alpha_{2}$ & -0.16 & -14.47 & -0.10 & -9.42 & -0.05 & -4.57 \\
$\alpha_{3}$ & 0.05 & 4.14 & 0.04 & 3.84 & -0.01 & -1.18 \\
$\alpha_{4}$ & 0.03 & 3.22 & 0.03 & 3.23 & 0.04 & 4.30 \\
\hline \hline
\end{tabular}

Table 6: Estimated Parameters of $\operatorname{AR}(p)$ for Essen, London Madrid 


\begin{tabular}{rrrrrrr}
\hline \hline & \multicolumn{2}{c}{ Paris } & \multicolumn{2}{c}{ Rome } & \multicolumn{2}{c}{ Stockholm } \\
& estimate & t.stat & estimate & t.stat & estimate & t.stat \\
\hline$\alpha_{1}$ & 0.91 & 106.98 & 0.82 & 97.09 & 0.90 & 106.36 \\
$\alpha_{2}$ & -0.19 & -16.99 & -0.09 & -8.12 & -0.19 & -16.70 \\
$\alpha_{3}$ & 0.07 & 8.23 & 0.03 & 3.63 & 0.10 & 11.46 \\
\hline \hline
\end{tabular}

Table 7: Estimated Parameters of $\operatorname{AR}(p)$ for Paris, Rome, Stockholm
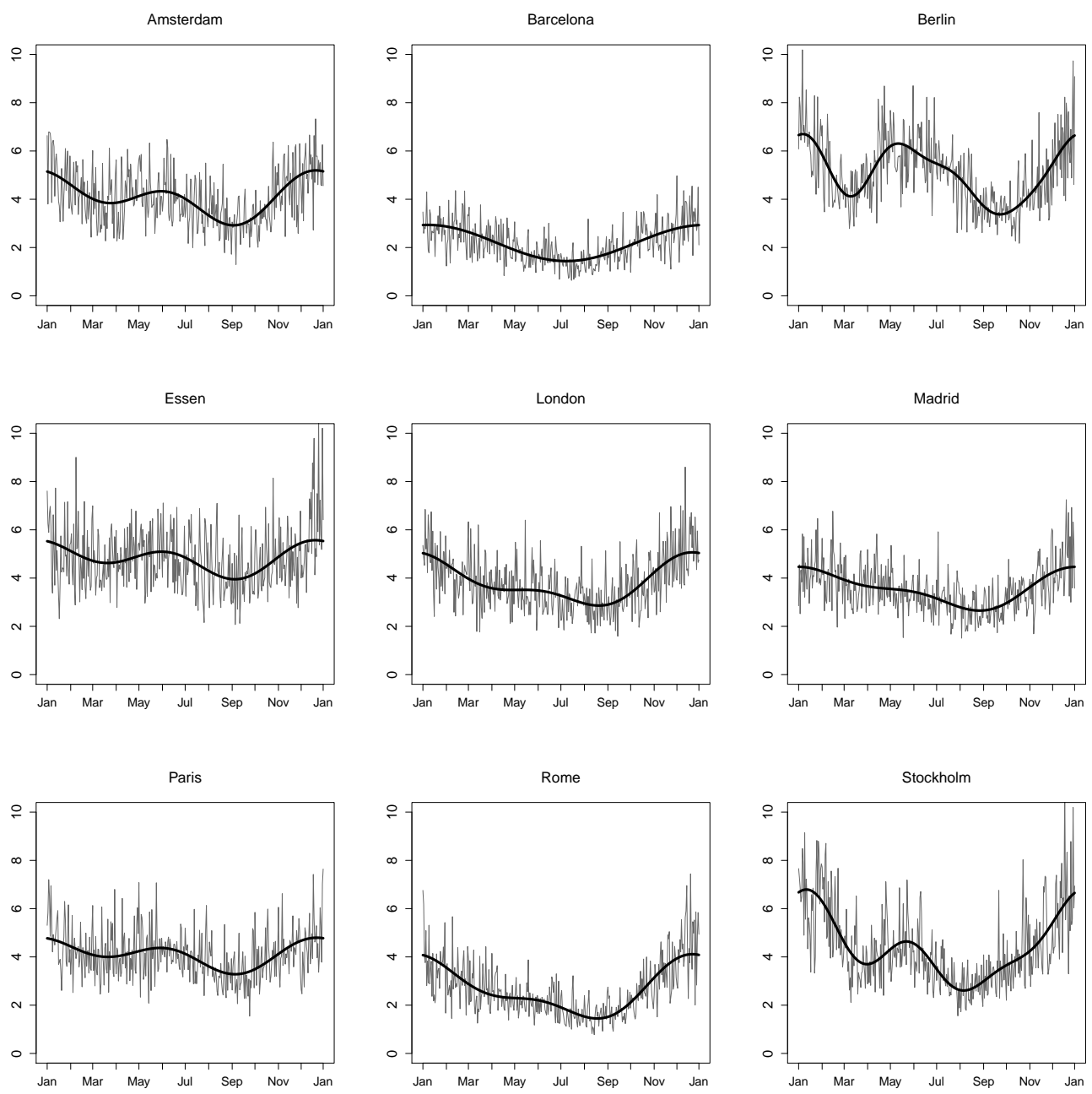

Figure 3: Estimated daily temperature variations (grey) and Fourier smoothed series (black). 

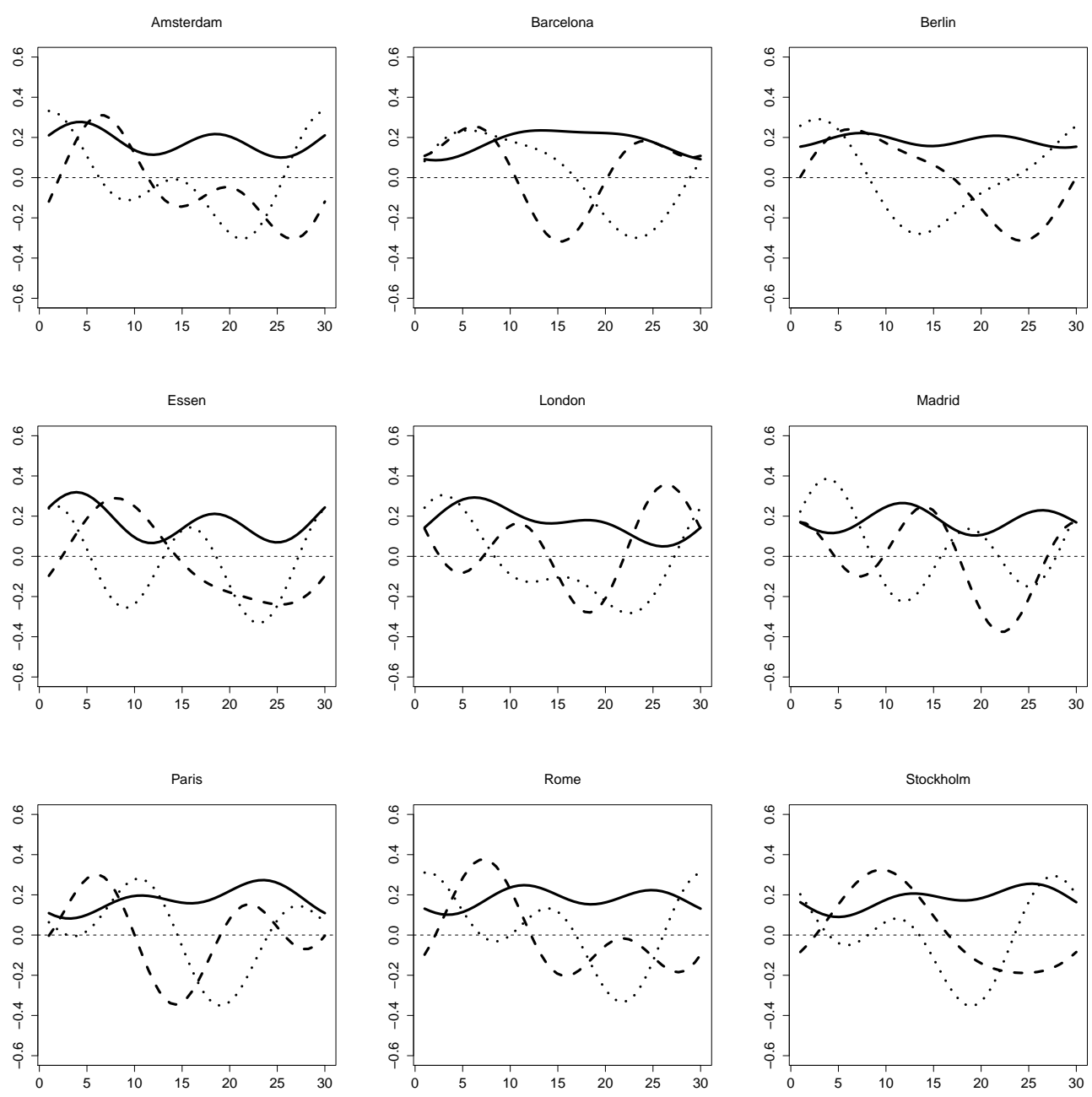

Figure 4: Eigenfunctions of temperature variation corresponding to the 3 largest eigenvalues of covariance operator

\subsection{GWR Model}

Now we consider our estimated risk premia and corresponding principal component scores in the geographically weighted regression. This local linear method allows for the capture of local patterns and heterogeneity in spatial 


\begin{tabular}{rcc}
\hline \hline City & $F_{C A T}(20100801,20100831)$ & $\widehat{F}_{C A T}(20100801,20100831, \theta=0)$ \\
\hline Amsterdam & 557 & 540 \\
Barcelona & 769 & 778 \\
Berlin & 607 & 573 \\
Essen & 570 & 577 \\
London & 594 & 577 \\
Madrid & 769 & 798 \\
Paris & 617 & 605 \\
Rome & 786 & 747 \\
Stockholm & 569 & 543 \\
\hline \hline
\end{tabular}

Table 8: Observed and estimated with $\theta=0 F_{C A T}$ prices for August 2010

risk premium generation, induced by temperature variation.

$$
\begin{aligned}
R P_{i j} & =\beta_{0}^{i}+\int \sum_{k=1}^{K} \beta_{k}^{i} \xi_{i k}(t)\left\{\sigma_{i j}(t)-\bar{\sigma}_{i}(t)\right\} d t+\varepsilon_{i j} \\
& =\beta_{i 0}+\sum_{k=1}^{3} \beta_{i k} c_{i k}+\beta_{i 4} d_{1}+\beta_{i 5} d_{2}+\tilde{\varepsilon}_{i j}, \quad \tilde{\varepsilon}_{i j} i i d .
\end{aligned}
$$

where $c_{i k}, k=1,2,3$ denote $k$ th PC score for $i$ th location, $i=1, \ldots, 9, d_{1}$ and $d_{2}$ are dummy variables to capture the effect of location on the north sea coast (London, Amsterdam) and south sea coast (Barcelona, Rome) respectively, and $\beta_{i 0}, \beta_{i 1}, \beta_{i 2}, \beta_{i 3}, \beta_{i 4}$ and $\beta_{i 5}$ are regression coefficients corresponding to the scores and dummy variables.

Following (15) we carry out the estimation. The estimated model parameters are shown in Table 9. The in-sample-fit is presented in Figure 5. Thereby the $R_{l o c}^{2}$ statistics measures how well the model calibrated at location $i$ can replicate the data at the vicinity of $i$. For some cities (Berlin, Rome, Stockholm) replication quality is rather high. For CAT futures on Amsterdam, Essen, London and Paris the model explains the low part of the variability, there it must be dominated by other factors rather than temperature variation. The $F$-test introduced by Fotheringham et al. (2002) to compare GWR with a global OLS model yields a $p$-value of 0.0061 , global model therefore can be rejected on $1 \%$ significance level.

We take Leipzig, Germany as an example to demonstrate a possible inter- 

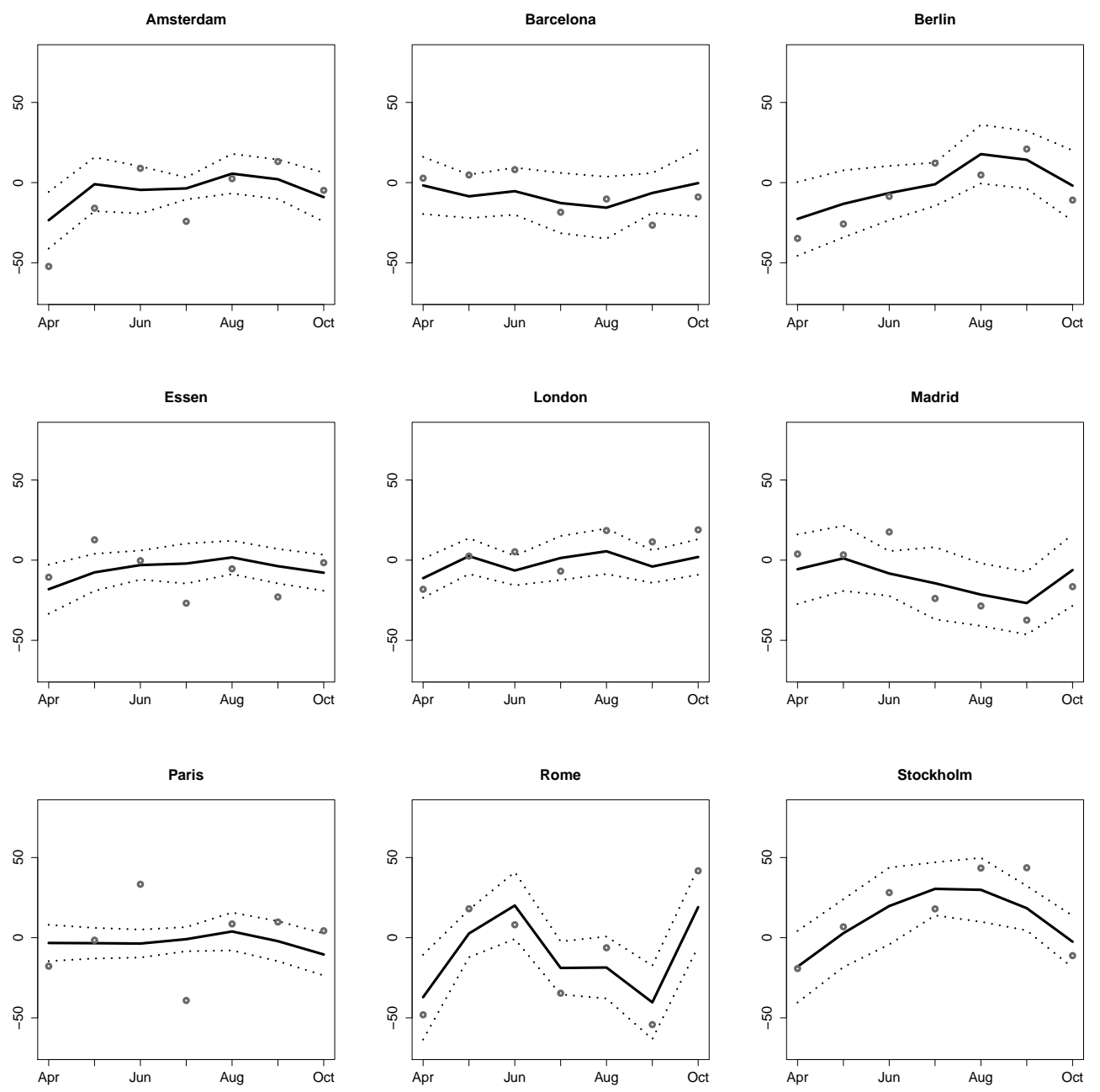

Figure 5: Risk premia (dots), fitted values by GWR (solid line) and 95\% CI

polation of risk premia to the locations in between the traded ones. We first, estimate seasonality $\Lambda_{t}$ and the dynamics of stochastic process $Y_{t}$, see Table 10. In a further step we find the eigenfunctions, bottom left plot in Figure 6, of the covariance operator and the resulting functional PC for $\sigma_{t}$ in Leipzig. Following (17) we find spatial coefficients $\beta$ using distance based weights for Leipzig and find prediction of risk premia using PC scores of Leipzig. Nonzero weights for prediction of risk premia in Leipzig were given to Berlin and Essen. The bottom right plot in Figure 6 shows predicted risk premia for Leipzig in comparison to the city with the highest weight (Berlin). Consequently, we are able to calculate $F_{C A T}$ prices, for Leipzig they would 


\begin{tabular}{rlrrrrrr}
\hline \hline City & $\beta_{0}$ & $\beta_{1}$ & $\beta_{2}$ & $\beta_{3}$ & $\beta_{4}$ & $\beta_{5}$ & $R_{\text {loc }}^{2}$ \\
\hline Amsterdam & $-4.68^{* *}$ & -5.28 & $15.31^{* *}$ & $15.04^{* *}$ & -0.30 & -3.39 & 0.25 \\
Barcelona & -7.35 & $6.17^{* *}$ & -5.53 & 8.27 & 5.77 & 0.03 & 0.34 \\
Berlin & -3.06 & -8.07 & $17.58^{* *}$ & $-9.90^{*}$ & -6.07 & -7.48 & 0.61 \\
Essen & $-5.10^{* *}$ & -4.86 & $14.87^{* *}$ & $10.85^{*}$ & -1.30 & -3.81 & 0.30 \\
London & -3.27 & -5.43 & $12.89^{* *}$ & $18.14^{* *}$ & 2.37 & -3.83 & 0.22 \\
Madrid & -10.40 & $11.17^{* *}$ & -7.98 & $30.55^{* *}$ & 12.36 & 3.42 & 0.42 \\
Paris & $-3.75^{* *}$ & -2.85 & $10.98^{*}$ & $13.38^{*}$ & 1.01 & -3.67 & 0.21 \\
Rome & $-5.54^{\circ}$ & $12.00^{*}$ & $-23.38^{* *}$ & $-77.01^{* *}$ & -2.83 & -4.85 & 0.73 \\
Stockholm & 10.82 & -16.29 & $16.91^{* *}$ & $-41.96^{* *}$ & $-20.79^{\circ}$ & -21.54 & 0.72 \\
\hline \hline
\end{tabular}

Table 9: Estimated Parameters of GWR. ${ }^{* *}$ indicate significance on $\leq 1 \%$ level, ${ }^{*}-$ on $5 \%$ and ${ }^{\circ}-$ on $10 \%$.

\begin{tabular}{rrr}
\hline \hline & estimate & t.stat \\
\hline$a$ & 8.75 & 134.36 \\
$b$ & $11 \cdot 10^{-5}$ & 10.67 \\
$c_{1}$ & -2.82 & -61.30 \\
$c_{2}$ & -8.92 & -193.82 \\
$c_{3}$ & 0.43 & 9.33 \\
$c_{4}$ & -0.01 & -0.29 \\
$c_{5}$ & -0.05 & -1.09 \\
$c_{6}$ & 0.24 & 5.18 \\
$c_{7}$ & 0.16 & 3.42 \\
\hline$\alpha_{1}$ & 0.97 & 115.26 \\
$\alpha_{2}$ & -0.27 & -23.63 \\
$\alpha_{3}$ & 0.11 & 12.76 \\
\hline \hline
\end{tabular}

Table 10: Estimated parameters of seasonality $\Lambda_{t}$ and $\operatorname{AR}(3)$ for Leipzig

be 322, 463, 495, 661, 536, 358 and 241 index points for April through to October 2010.

\subsection{Hedging Weather Exposure in Electricity}

As already pointed out in the introduction, electric utilities are exposed to weather risk. Although many other hedging instruments like power futures are available, they are primarily suitable for hedging price and cost risks, not quantity risk. Temperature derivatives on the contrary are designed to 

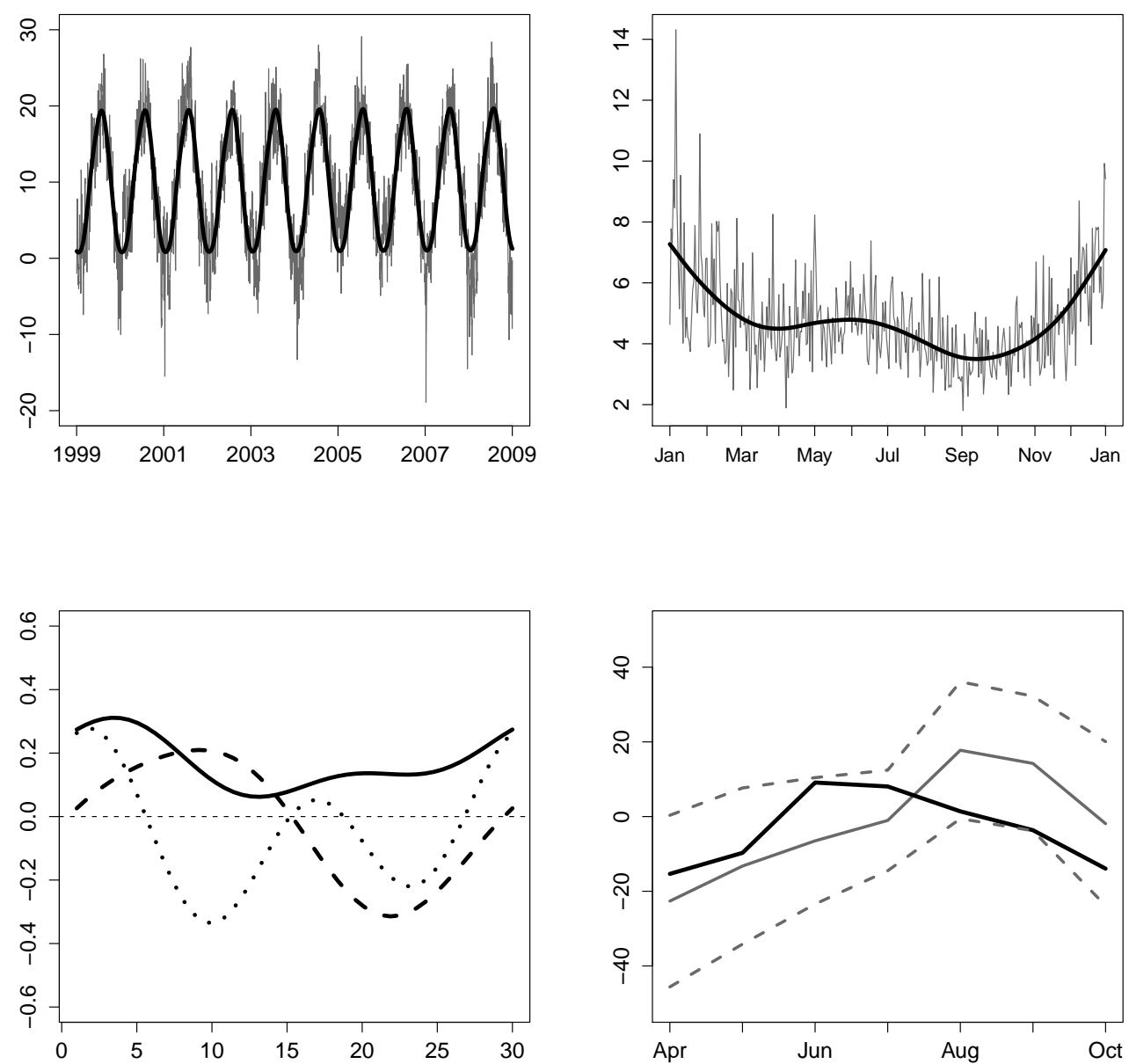

Figure 6: Temperature seasonality (top left), seasonal variation (top right), eigenfunctions of temperature variation for Leipzig (bottom left), left plot and predicted risk premium (black line) in comparison to risk premia of Berlin (grey), bottom right

hedge the quantity risks faced by the energy sector. An example would be an electricity utility exposed to high costs of meeting additional demand on eletricity during unusually hot summers. With WD available, the utility may enter a futures contract on CAT for the summer months. If the summer is hot it will receive a reimbursement for the extra costs. In this section we consider an electricity utility, which is supposed to face weather dependent demand as in Figure 7 . 


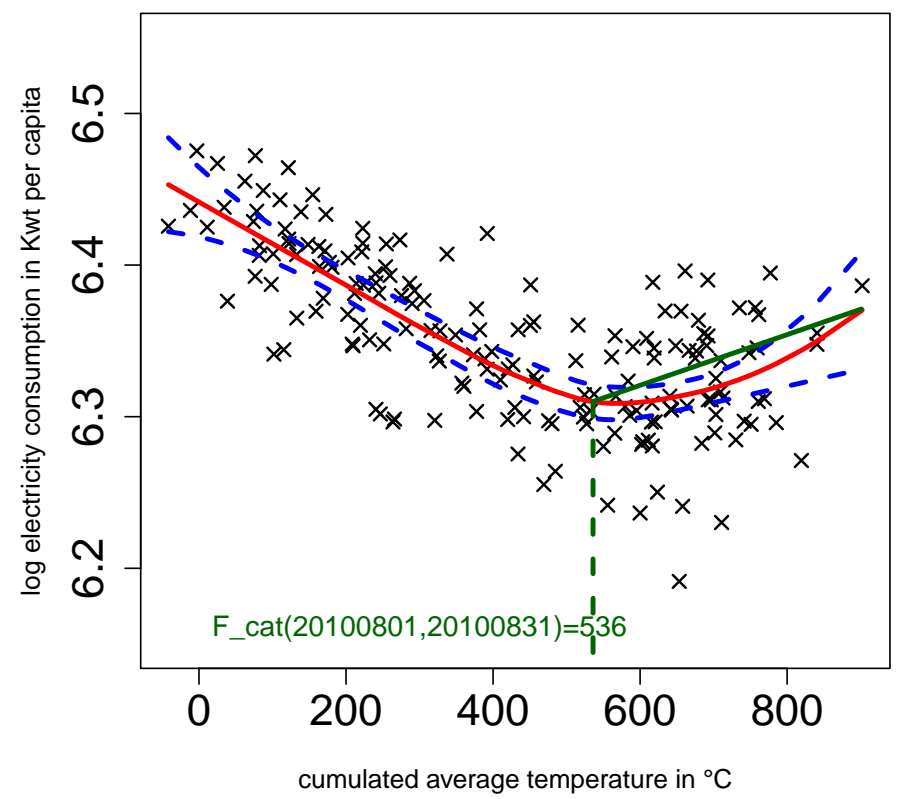

Figure 7: Illustration of hedging strategy with August CAT future price $536^{\circ}$ C estimated from the model

We now can use the results from the previous sections to study an example of a hedging strategy for an electricity provider in Leipzig. Using the estimated risk premia for Leipzig we can obtain the $F_{C A T}$ price, e.g. for August 2010 $\widehat{F}_{C A T}=536^{\circ}$ based on historical data. Let $c$ be the marginal cost of meeting additional log demand of $1 \%$ per person, $b$ is the estimated marginal effect of $1^{\circ} \mathrm{C}$ CAT on log demand starting from threshold $F_{C A T}$, here it is the slope of the solid green line in Figure (7). If $\alpha$ is the number of the WD hold and $t$ - tick value of the WD (for traded futures on continental Europe - 20 EUR, UK - 20 GBP). Then, the exposure to temperature caused fluctuations in demand is $\approx c b\left(C A T-F_{C A T}\right)$ and the possible benefit of holding $\alpha$ temperature futures is $\alpha t\left(C A T-F_{C A T}\right)$. Hedging under no transaction costs is enhanced by buying $\alpha^{*}$ CAT futures such that holds $c b=\alpha^{*} t$.

Other hedging possibilities are described in Leggio and Lien (2002). The results presented above can be extended straight forwardly to risk premia on other kinds of temperature futures and therefore enable the hedging of weather sensitive demands in different spatial points. 


\section{Conclusion}

We examined spatial risk premia on European CAT futures at the start of the measurement period. By means of geographically weighted regression we quantified the effect of temperature variation on the risk premia. Our results showed that temperature variation explains significant proportions of the variation in risk premia. With our model we were able to locally predict variation induced risk premium as well as the future price for other locations and execute hedging strategies; this was illustrated using the example of Leipzig, Germany. 


\section{References}

Akdeniz-Duran, E., Härdle, W.K., And M. Osipenko (2011): "Difference-based Ridge and Liu-type Estimators in Semiparametric Regression Models," SFB 649 Discussion Paper 2011-000.

Alaton, P., B. Djehiche, And D. Stillberger (2002): "On Modelling and Pricing Weather Derivatives," Appl. Math. Finance, 1, 1-20.

Asche, F., O. Nilsen, And R. Tveteras (2008): "Natural Gas Demand in the European Household Sector," The Energy Journal, 29, 27-46.

Benth, F., J. Saltyte Benth, and S. Koekebakker (2007): "Putting a Price on Temperature," Scandinavian Journal of Statistics, 747-767.

Box, G. And G. Jenkins (1970): Time Series Analysis - Forecasting and Control, San Francisco: Holden Day.

Campbell, S. And F. Diebold (2005): "Weather Forecasting for Weather Derivatives," American Stat. Assoc., 469, 6-16.

Chaumont, S. Imkeller, P. And M. Müller (2006): "Equilibrium Trading of Climate and Weather Risk and Numerical Simulation in a Markovian Framework," Stoch. Environ. Res. Risk Assess., 184-205.

Considine, G. (2000): "Introduction to Weather Derivatives," .

Davis, M. (2001): "Pricing Weather Derivatives by Marginal Value," Quantitative Finance, 1, 305-308.

Dornier, F. And M. Querel (2000): "Caution to the Wind," Energy Power Risk Management, in: Weather Risk Special Report, 30-32.

Fotheringham, A., C. Brudson, and M. Charlton (2002): Geographically Weigthed Regression: the Analysis of Spatially Varying Relationships, John Willey \& Sohns.

Gillespie, D. (1996): "Exact numerical simulation of the OrnsteinUhlenbeck process and its integral," Physical Review, 2084-2091.

Härdle, W. And B. López Cabrera (2011): "Implied Market Price of Weather Risk," Applied Math. Finance (tentativ. accepted).

Horst, U. AND M. MÜller (2007): "On the spanning property of risk bonds priced by equilibrium," Mathematics of Operation Research, 784807. 
Hurvich, C. And C. Tsai (1989): "Regression and time series model selection in small samples," Biometrica, 297-307.

LeE, Y. AND S. Oren (2009): "An equilibrium pricing model for weather derivatives in a multi-commodity setting," Energy Economics, 31, 702-713.

LegGio, K. And D. Lien (2002): "Hedging gas bills with weather derivatives," Journal of Economics and Finance, 88-100.

Metcalf, G. (2008): "An empirical analysis of energy intensity and its determinants at the state level," The Energy Journal, 29, 1-26.

ØKsendal, B. (2000): Stochastic Differential Equations, New York: Springer.

Perez-Gonzalez, F. And H. Yun (2010): "Risk Management and Firm Value: Evidence from Weather Derivatives," Available at SSRN: http://ssrn.com/abstract $=1357385$.

Ramsay, J. And B. Silverman (1997): Functional Data Analysis, New York: Springer.

Rosenthal, D. H., H. K. Gruenspecht, and E. A. Moran (1995): "Effects of Global Warming on Energy Use for Space Heating and Cooling in the United States," The Energy Journal, 16, 77-96. 


\section{SFB 649 Discussion Paper Series 2011}

For a complete list of Discussion Papers published by the SFB 649, please visit http://sfb649. wiwi. hu-berlin.de.

001 "Localising temperature risk" by Wolfgang Karl Härdle, Brenda López Cabrera, Ostap Okhrin and Weining Wang, January 2011.

002 "A Confidence Corridor for Sparse Longitudinal Data Curves" by Shuzhuan Zheng, Lijian Yang and Wolfgang Karl Härdle, January 2011.

003 "Mean Volatility Regressions" by Lu Lin, Feng Li, Lixing Zhu and Wolfgang Karl Härdle, J anuary 2011.

004 "A Confidence Corridor for Expectile Functions" by Esra Akdeniz Duran, Mengmeng Guo and Wolfgang Karl Härdle, January 2011.

005 "Local Quantile Regression" by Wolfgang Karl Härdle, Vladimir Spokoiny and Weining Wang, January 2011.

006 "Sticky Information and Determinacy" by Alexander Meyer-Gohde, January 2011.

007 "Mean-Variance Cointegration and the Expectations Hypothesis" by Till Strohsal and Enzo Weber, February 2011.

008 "Monetary Policy, Trend Inflation and Inflation Persistence" by Fang Yao, February 2011.

009 "Exclusion in the All-Pay Auction: An Experimental Investigation" by Dietmar Fehr and Julia Schmid, February 2011.

010 "Unwillingness to Pay for Privacy: A Field Experiment" by Alastair R. Beresford, Dorothea Kübler and Sören Preibusch, February 2011.

011 "Human Capital Formation on Skill-Specific Labor Markets" by Runli Xie, February 2011.

012 "A strategic mediator who is biased into the same direction as the expert can improve information transmission" by Lydia Mechtenberg and J ohannes Münster, March 2011.

013 "Spatial Risk Premium on Weather Derivatives and Hedging Weather Exposure in Electricity" by Wolfgang Karl Härdle and Maria Osipenko, March 2011. 